\title{
Alitretinoin for the treatment of severe chronic hand eczema
}

\author{
This article was published in the following Dove Press journal: \\ Patient Preference and Adherence \\ 25 November 2014 \\ Number of times this article has been viewed
}

\section{Thomas King \\ John McKenna}

Anton B Alexandroff

Department of Dermatology, University Hospitals of Leicester, Leicester Royal Infirmary, Leicester, UK
Correspondence: Anton B Alexandroff Department of Dermatology, Leicester Royal Infirmary, Infirmary Square, Leicester LEI 5WW, UK

Email anton.alexandroff@uhl-tr.nhs.uk
Abstract: Chronic hand eczema is a common and often debilitating condition. Alitretinoin, a 9-cis-retinoic acid and pan-retinoic acid agonist, is a new and effective systemic treatment for chronic hand eczema, which provides another treatment option. A "clear" or "almost clear" response can be achieved in up to half of patients within a 24-week course of treatment. Even higher rates of remission can be obtained with a longer duration of treatment. Alitretinoin has a favorable overall profile of adverse effects; however, female patients who are at risk of becoming pregnant should follow a strict pregnancy-prevention program due to the teratogenic effects of this drug.

Keywords: dermatitis, toctino, CTCL

\section{Introduction}

Eczema or dermatitis are broad terms to describe an inflammatory skin condition, which may be dry, red, itchy, and flaky. Dermatitis is the most common skin disease affecting the hands. Chronic hand eczema (CHE) affects $2 \%-9 \%$ of the population and up to $30 \%$ of people in risk professions, such as health care workers, hairdressers, cleaners, cooks, or those working in manufacturing industries. Severe CHE can be a truly devastating condition that can affect patients' quality of life to the same extent as asthma and psoriasis. ${ }^{1}$ Dermatitis also has a major economic impact, and may lead to a significant loss of productivity. ${ }^{2}$

A number of causes of $\mathrm{CHE}$ are recognized, which are often multifactorial. These include atopic dermatitis, irritant contact dermatitis, and allergic contact dermatitis. ${ }^{2}$ There are a number of common allergens, including metals such as nickel and chromium, rubber additives, plastics, resins, biocides, preservatives, and fragrances. Common irritants include organic solvents, detergents, and water itself. It should be remembered that both irritant and allergic contact dermatitis are more common in atopic individuals.

There are many different subtypes of hand eczema, with varying clinical presentations, which can make a precise diagnosis challenging. In addition to the classification based on etiology, CHE can be classified depending on localization and morphology; the latter includes hyperkeratotic and pompholyx variants. Overall, seven distinct subdiagnoses have been agreed upon in a recent European study. ${ }^{3}$

\section{Diagnosis}

Diagnosis of CHE is usually clinical, and is based on history, including personal and family history of atopy, occupational factors, hobbies, and skin-care products used. 
Physical examination should include thorough inspection of appearance and distribution of dermatitis on the hands, as well as affected areas elsewhere on the body. In particular, feet should be carefully examined, as feet are often affected in patients with different types of CHE.

Important investigations to consider are bacterial swabs for culture to look for secondary infection. Fungal skin scrapings should be considered if the feet are involved or if only one hand is affected. Differential diagnosis may include psoriasis, dermatophyte infections, scabies, lichen planus, dermatomyositis, pityriasis rubra pilaris, and rarely mycosis fungoides. If diagnosis is in question, a skin biopsy may be considered. Patch testing is recommended if an allergic contact dermatitis of delayed type IV hypersensitivity reactions is suspected. The European Standard Series ${ }^{4}$ is most commonly used in Europe and elsewhere in the world, but the North American Series ${ }^{5}$ is used in the US. In addition, products should be tested if appropriate. If allergic contact dermatitis of type I hypersensitivity reactions is assumed, radioallergosorbent or skin-prick tests are employed.

\section{Standard treatments}

As with most chronic diseases, there is a stepwise approach to management. Basic skin care is the cornerstone of management, which includes avoidance of common irritants, discontinuing skin-care products that may be an irritant (eg, aqueous cream), and use of personal protective equipment, such as gloves. Gloves made of plastic or polyvinyl chloride material are preferable to rubber in order to avoid accelerator chemicals. Hands should be kept well moisturized with frequent application of a suitable emollient, especially after washing hands. Moisturizing soap substitutes should be routinely used, and ointments are preferred to creams and lotions in this setting.

It is quite important to diagnose whether the patient is suffering from irritant contact dermatitis or allergic contact dermatitis, and in the latter it is imperative to determine the precise relevant allergen(s) involved. The patient should be meticulously educated to avoid such allergen(s) in future, because even very small quantities of these substances can trigger allergic contact dermatitis.

Topical corticosteroids are used as the next step up. As with emollients, ointments are preferred over creams, due to increased absorption and efficacy. Moderately potent topical corticosteroids are routinely used, but often the strength of topical corticosteroids has to be upgraded to potent and very potent preparations. Furthermore, application under occlusion makes topical corticosteroids more effective.
Unfortunately, severe $\mathrm{CHE}$ is often resistant to even very potent topical corticosteroids, and side effects with longerterm use, such as skin atrophy, limit their use for long-term treatment. Topical calcineurin inhibitors (tacrolimus ointment or pimecrolimus cream) can be used as maintenance therapy, but generally they are more effective when used on face and skin folds areas compared to hands and feet. ${ }^{2}$

Phototherapy is reserved as second-line treatment for CHE. Psoralen ultraviolet A (PUVA) has been shown to be superior to UVB. ${ }^{6}$ Grenz ray, a type of superficial ionizing radiation, has also been used to treat hand dermatitis. Although high-dose Grenz irradiation has been associated with the development of nonmelanoma skin cancers, Grenz-ray therapy is still considered by some authorities as a safe treatment modality when administered according to the guidelines. ${ }^{7}$

If the aforementioned measures are not sufficient, systemic therapy should be considered. Short-term courses of corticosteroids are sometimes used for acute flare-ups, although their side-effect profiles make them unsuitable for long-term use. Notably, oral corticosteroids usually act faster than topical corticosteroids, and thus may be very useful when it is important to bring severe $\mathrm{CHE}$ under control quickly. A number of steroid-sparing systemic agents have been used for hand eczema, including azathioprine, cyclosporine, and methotrexate; however, the only systemic therapy currently licensed for use for $\mathrm{CHE}$ is alitretinoin. ${ }^{8-11}$ It should be noted that azathioprine, methotrexate, and cyclosporine, in contrast to alitretinoin, are immunosuppressive medications, and if used long term may increase the risk of skin cancers. All three drugs may also cause bone marrow suppression, and in addition azathioprine and methotrexate are hepatotoxic, whereas cyclosporine may cause irreversible renal impairment. Furthermore, methotrexate is a teratogenic medication.

\section{Alitretinoin \\ Mechanism of action/pharmacodynamics}

Alitretinoin (9-cis-retinoic acid) is an endogenous retinoid related to vitamin $\mathrm{A}$. It binds to intracellular retinoic acid receptors $A(R A R)$ and $X(R X R)$. In contrast, isotretinoin and acitretin bind selectively to RAR, while bexarotene is selective for RXR. This distinction may explain why alitretinoin is effective in control of $\mathrm{CHE}$ while other retinoids (isotretinoin, acitretin, bexarotene) are not. The assumption is that binding to both receptors is required for control of CHE.

Although the exact mechanism of action of alitretinoin in $\mathrm{CHE}$ is yet to be elucidated, it is believed to involve the regulation of the expression of genes that control cellular 
differentiation and proliferation. It has also been shown to have immunomodulatory and anti-inflammatory effects by chemokine-induced leukocyte recruitment and inhibition of dendritic cell-mediated T-cell activation. ${ }^{12}$

\section{Side effects and patient safety}

Most side effects of alitretinoin have been shown to be reversible, dose-dependent, and consistent with side effects for other retinoids. The standard dose of alitretinoin is $30 \mathrm{mg}$ once daily (OD), reducing to $10 \mathrm{mg}$ OD if intolerable side effects are encountered. A dose of $10 \mathrm{mg}$ OD from the beginning may be considered in at-risk patients, eg, patients with diabetes mellitus. The most common side effect of alitretinoin is headache. ${ }^{13}$ Headache can usually be controlled by simple painkillers, but in some patients it may necessitate discontinuation of the drug. Sensitivity to sunlight is a common problem, and patients should avoid excessive sunlight, use high sun protection-factor sun creams, and avoid sun beds. Dryness of the lips, skin, and mouth, anemia, flushing, and erythema are the other most frequent adverse effects. However, dryness of the lips and skin appears to be a very rare side effect. The National Health Service website indicates that it is seen "more than $1 / 100$ ", although that seems not too different than the general population. Most clinicians will agree that dry skin and lips are not a problem with this drug (unlike acitretin and isotretinoin). Decreased night vision has been reported. No psychiatric adverse effects have been found. Increases in serum cholesterol and triglycerides and a decrease in serum thyroid-stimulating hormone sometimes occur, but to date have not led to discontinuation of alitretinoin in the setting of clinical trials. ${ }^{13}$

Alitretinoin, like other retinoids, is teratogenic. Female patients of a childbearing age who are at risk of becoming pregnant should be clearly counseled and started on a pregnancy-prevention program prior to commencement of alitretinoin. At least one reliable method of contraception, ideally combined with a second method of contraception, should be commenced 1 month before starting alitretinoin and continued without interruptions during the whole period of treatment and for 1 month after stopping alitretinoin. Pregnancy testing should be done prior to the commencement of alitretinoin and subsequently every month during the treatment, as well as 1 month after discontinuation of the medication. Prescriptions should be limited to 30 days of treatment, pregnancy testing should happen on the same day as prescribing, and dispensing should happen within a maximum of 7 days of the prescription being issued. Patients should not give blood during and for 1 month after their treatment, due to the risk of the recipient being a pregnant female. Overall, because of potential side effects, the ideal target population for alitretinoin might be nonatopic nonobese male patients with CHE.

\section{Drug interactions}

Alitretinoin is metabolized by cytochrome p450 3A4 (CYP3A4). ${ }^{14}$ Therefore, CYP3A4 inhibitors, such as ketoconazole, can raise the plasma levels of alitretinoin. In addition, alitretinoin reduces the plasma concentration of simvastatin. In practice however, the effects of the interrelations appear to be small. ${ }^{14}$ Vitamin A supplements and other retinoids should not be taken in conjunction with alitretinoin, due to the risk of hypervitaminosis A. Drug interactions with hormonal contraceptives should also be taken into account if they are being used. Due to the known interaction of other retinoids with tetracyclines causing benign intracranial hypertension, tetracyclines should not be used in combination with alitretinoin.

\section{Cost}

Alitretinoin costs $£ 411.43$ for a pack of $30 \times 30 \mathrm{mg}$ capsules. Therefore, a 6-month treatment course costs $£ 2,468.58$.

\section{Evidence of efficacy}

The Benefit of Alitretinoin in Chronic Hand Dermatitis $(\mathrm{BACH})$ study was the first Phase III trial of alitretinoin. ${ }^{13}$ It was a randomized, double-blind, multicenter, placebocontrolled trial that investigated the safety and efficacy of alitretinoin. A total of 1,032 patients with severe refractory CHE in 111 dermatology outpatient clinics were randomized in a 1:2:2 ratio to placebo, $10 \mathrm{mg}$, or $30 \mathrm{mg}$ OD oral alitretinoin for up to 24 weeks. All patients were told to apply an emollient frequently, but no other medication for CHE was allowed. All responders were followed up for a further 24 weeks to assess relapse, when no additional treatment was allowed. The primary endpoint of the study was to assess response to treatment, with satisfactory responses being either "clear" or "almost clear" on the physician global assessment (PGA) of overall CHE-severity tool. The trial clearly demonstrated that up to $48 \%$ of patients achieved clear or almost-clear status compared with $17 \%$ for placebo $(P<0.001)$, with up to $75 \%$ median reduction in disease signs and symptoms. The response was dose-dependent (Table 1). The response was evident after 2 months of treatment, and reached a plateau after 6 months of treatment. Patients with all types of eczema responded; however, the response varied depending on the type of CHE: hyperkeratotic 49\%, fingertip 
Table I Response of patients with chronic hand dermatitis to alitretinoin in the Benefit of Alitretinoin in Chronic Hand Dermatitis study

\begin{tabular}{|c|c|c|c|}
\hline & \multicolumn{3}{|c|}{$\begin{array}{l}\text { Alitretinoin dose (intention-to-treat } \\
\text { population) }\end{array}$} \\
\hline & $\begin{array}{l}10 \mathrm{mg} \\
(\mathrm{n}=4 \mid 8)\end{array}$ & $\begin{array}{l}30 \mathrm{mg} \\
(\mathrm{n}=409)\end{array}$ & $\begin{array}{l}\text { Placebo } \\
(n=205)\end{array}$ \\
\hline \multicolumn{4}{|c|}{ Physician global assessment } \\
\hline Clear & $39(9.3 \%)$ & 90 (22.0\%) & $6(2.9)$ \\
\hline Almost clear & 76 (I8.2\%) & $105(25.7)$ & $28(13.7 \%)$ \\
\hline Clear or almost clear & II 5 (27.5\%) & 195 (47.7\%) & $34(16.6 \%)$ \\
\hline Comparison to placebo & $P=0.004$ & $P<0.00$ I & NA \\
\hline
\end{tabular}

Abbreviation: NA, not applicable.

$44 \%$, and pompholyx 33\% (the response was defined as clear or almost clear PGA, and the categories for type of $\mathrm{CHE}$ were not mutually exclusive). The median time to relapse, defined as recurrence of $75 \%$ of initial signs and symptoms, was 5.5 months in the absence of antieczema medication. However, two-thirds of patients who achieved clear or almostclear status remained in remission 6 months later.

A second randomized double-blind placebo-controlled trial was carried out in 117 patients with severe CHE who had previously responded to treatment in the BACH study and who had relapsed within the 24-week follow-up period. ${ }^{15}$ Patients who had originally been treated with alitretinoin were randomized to either their previous alitretinoin dose or placebo in a 2:1 ratio for 12-24 weeks. Patients who had responded to placebo in the $\mathrm{BACH}$ trial were allocated to the placebo group again. As in the BACH study, patients used emollients regularly, and no other treatments for CHE were allowed. Similarly to the BACH study, the outcome of the trial was defined as PGA "clear" or "almost clear". Overall results showed that retreatment was well tolerated and effective, with highest response rates seen with a $30 \mathrm{mg}$ dose and adverse events similar to those in the first study.

An open-label trial was also carried out to study an extended treatment with a further 12-24 week course of oral alitretinoin $30 \mathrm{mg}$ in patients who did not respond to initial therapy in the BACH trial. ${ }^{16}$ At the end of the BACH study, patients whose eczema was graded as mild, moderate, or severe according to their PGA were eligible for this trial. All 243 patients received $30 \mathrm{mg}$ alitretinoin, irrespective of their treatment in the BACH trial. In this extendedtreatment trial, ${ }^{16}$ the PGA response rate was $50 \%$ and $39 \%$ in patients who had taken $10 \mathrm{mg}$ and $30 \mathrm{mg}$ alitretinoin, respectively, in the initial $\mathrm{BACH}$ trial. This study demonstrated that a significant proportion of patients who initially failed to respond to either 10 or $30 \mathrm{mg}$ of alitretinoin could still benefit from an extended course of treatment for a further 12-24 weeks. In this trial, alitretinoin was found to be well tolerated for up to 48 weeks.

The aforementioned double-blind Phase III trials showed alitretinoin to be well tolerated and effective in the treatment of CHE. However, because these trials were double-blinded, it was not possible to use a flexible dose to minimize adverse effects, as would usually happen in clinical practice. An open-label study assessing the safety and efficacy of alitretinoin in patients with severe CHE unresponsive to topical corticosteroids studied 249 patients with severe CHE unresponsive to topical corticosteroids. ${ }^{17}$ They were given alitretinoin $30 \mathrm{mg}$ OD initially for up to 24 weeks, but dose reduction occurred in $16.5 \%$ patients and dose interruption in $15.7 \%$ of patients, respectively, due to adverse effects or changes in monitoring tests. A PGA of "clear" or "almost clear" was reported in $46.6 \%$ of patients, which was similar to the aforementioned blinded trials. Results of a visual analog scale and a categorical scale for pruritus also provided supportive evidence for alitretinoin efficacy.

The TOCCATA (Toctino ${ }^{\circledR}$ in severe chronic hand eczematherapy in an observational study) noninterventional observational open study was designed to investigate the use of alitretinoin in the treatment of CHE under "reallife" medical practice conditions in Germany. ${ }^{18}$ A total of 680 patients were treated with alitretinoin as per the summary of product characteristics for up to 24 weeks. Again, the PGA, defined as "clear" or "almost clear", was the primary outcome assessment of the response. In total, $56.7 \%$ of patients responded to the treatment, with only a small variation within different morphological forms. Hyperkeratotic CHE showed the highest response rate $(59.2 \%)$, followed by fingertip dermatitis $(52.2 \%)$ and vesicular CHE (47.9\%).

\section{NICE guidelines}

The National Institute for Health and Care Excellence (NICE) technology-appraisal guidance for treatment of severe CHE with alitretinoin was published in August 2009. ${ }^{19}$ Alitretinoin is recommended by NICE for use in severe CHE that has not responded to potent topical steroids. The severity of CHE is defined as severe on the PGA in combination of a dermatology quality-of-life index score of 15 or more. NICE recommends to stop treatment if 1 ) there is an adequate response, 2) there is still severe disease at 12 weeks of treatment, or 3 ) an adequate response has not been achieved by 24 weeks. Alitretinoin should be prescribed only by dermatologists or physicians with experience of managing $\mathrm{CHE}$ and monitoring systemic retinoids. 


\section{Use of alitretinoin off license}

Alitretinoin has also been reported to be helpful in other dermatological conditions beyond CHE. A case series of seven patients showed that alitretinoin may be effective to treat palmoplantar pustular psoriasis. ${ }^{20-21}$ Three small case series provided initial evidence that alitretinoin may also be effective in the treatment of cutaneous T-cell lymphoma. ${ }^{22-24}$ A larger controlled study is required to confirm these initial findings. According to case reports, systemic alitretinoin may also be effective in lichen planus, ${ }^{25}$ Hailey-Hailey disease, ${ }^{26}$ pityriasis rubra pilaris, ${ }^{27}$ Darier disease, ${ }^{28-29}$ alopecia areata, ${ }^{30}$ and dissecting cellulitis of the scalp. ${ }^{31}$

\section{Conclusion}

CHE is a common skin condition that can be distressing and debilitating for a significant proportion of patients. While good hand care, frequent use of emollients, and a judicious use of topical steroids are essential part of its management, systemic treatment is necessary in a number of patients. Patch testing should be considered in patients who fail potent topical corticosteroids. PUVA photochemotherapy may be effective, but it is often difficult for patients to receive, due to a necessity to travel to hospital. In addition, CHE often relapses quickly after PUVA. Older immunosuppressive treatments (cyclosporine, azathioprine, methotrexate) may be effective, but have potentially life-threatening adverse effects, including bone marrow suppression and renal and hepatic impairment. Alitretinoin, a 9-cis-retinoic acid pan-retinoic acid-receptor agonist is the only currently licensed systemic medication for CHE. "Clear" or "almost clear" status can be achieved in half of the patients treated for up to 24 weeks. A further response of between $39 \%$ and $50 \%$ can be achieved if the course of treatment is extended for another 12-24 weeks. Alitretinoin has overall a favorable side-effect profile, and provides another treatment option. However, as with all retinoids, alitretinoin is highly teratogenic, and female patients who are at risk of becoming pregnant should follow a strict pregnancy prevention-program similar to that of isotretinoin. ${ }^{32}$

\section{Disclosure}

ABA is the lead of the LNR Comprehensive Local Research Networks Dermatology Specialty Group, a member of the Biologics for Psoriasis Industry Subgroup, and the Steering Group of the UK Dermatology Clinical Trial Network. He has received fellowships, educational grants, consultancy fees, and acted as an investigator or a member of advisory board or a director for Abbott Laboratories, Novartis, Pfizer, Procter and Gamble, Merck Serono, LEO Pharma, Basilea Pharmaceutica,
Apodi, Genus Pharmaceuticals, GlaxoSmithKline, Medefield, EMS Research, CSD Health Research, XBioCell, Bryter Research, Genactis, Medicus, Research House, All Global, Keyquest, Teva, Almirall, and Galderma. JM is the deputy lead of the LNR Comprehensive Local Research Networks Dermatology Specialty Group; he has received educational grants, consultancy fees, and acted as an investigator for Novartis, Galderma, Abbott, Leo Pharma, Genus Pharmaceuticals, and Almirall. TK reports no conflicts of interest.

\section{References}

1. English J, Graham-Brown R, de Sica CA, Alexandroff AB. Everyday clinical experience of alitretinoin in the treatment of severe chronic hand eczema: seven case studies. Clin Exp Dermatol. 2011; Suppl 36:1-2

2. Alexandroff AB, Johnston GA. Medical management of contact dermatitis. G Ital Dermatol Venereol. 2009;144:537-540.

3. Diepgen TL, Andersen KE, Brandao FM, et al. Hand eczema classification: a cross-sectional, multicentre study of the aetiology and morphology of hand eczema. Br J Dermatol. 2009;160: 353-358.

4. Castelain M, Assier H, Baeck M et al. The European Standard Series and its additions: are they of any use in 2013? Eur J Dermatol. 2014 Jan-Feb;24(1):15-22.

5. Schalock PC, Dunnick CA, Nedorost S et al. American contact dermatitis society core allergen series. Dermatitis. 2013 Jan-Feb;24(1):7-9.

6. Rosen K, Mobacken H, Swanbeck G. Chronic eczematous dermatitis of the hands: a comparison of PUVA and UVB treatment. Acta Derm Venereol. 1987;67:48-54.

7. Warner JA, Cruz PD Jr. Grenz ray therapy in the new millennium: still a valid treatment option? Dermatitis. 2008;19:73-80

8. Granlund H, Erkko P, Eriksson E, Reitamo S. Comparison of cyclosporine and topical betamethasone-17,21-dipropionate in the treatment of severe chronic hand eczema. Acta Derm Venereol. 1996;76:371-376.

9. Berth-Jones J, Takwale A, Tan E, et al. Azathioprine in severe adult atopic dermatitis: a double-blind, placebo-controlled, crossover trial Br J Dermatol. 2002;147:324-330.

10. Meggitt SJ, Gray JC, Reynolds NJ. Azathioprine dosed by thiopurine methyltransferase activity for moderate-to-severe atopic eczema: a double-blind, randomised controlled trial. Lancet. 2006;367: 839-846.

11. Weatherhead SC, Wahie S, Reynolds NJ, Meggitt SJ. An open-label, dose-ranging study of methotrexate for moderate-to-severe adult atopic eczema. Br J Dermatol. 2007;156:346-351

12. Levin AA, Sturzenbecker LJ, Kazmer S, et al. 9-cis Retinoic acid stereoisomer binds and activates the nuclear receptor RXR alpha. Nature. 1992;355:359-361.

13. Ruzicka T, Lynde CW, Jemec GB, et al. Efficacy and safety of oral alitretinoin (9-cis retinoic acid) in patients with severe chronic hand eczema refractory to topical corticosteroids: results of a randomized, double-blind, placebo-controlled, multicentre trial. Br J Dermatol. 2008;158:808-817.

14. Schmitt-Hoffmann AH, Roos B, Sauer J, et al. Pharmacokinetic interactions between alitretinoin and ketoconazole or simvastatin or ciclosporin A. Clin Exp Dermatol. 2011;36 Suppl 2:24-28.

15. Coenraads PJ, Ruzicka T, Lynde C, et al. Alitretinoin re-induces response in relapsed CHE patients. Poster presented at: 9 th Biennial Congress of the European Society of Contact Dermatitis; May 28-31, 2008; Lisbon, Portugal.

16. Lynde C, Cambazard F, Ruzicka T, Sebastian M, Brown TC, Maares J. Extended treatment with oral alitretinoin for patients with chronic hand eczema not fully responding to initial treatment. Clin Exp Dermatol. 2012;37:712-717. 
17. Dirschka T, Reich K, Bissonnette R, Maares J, Brown T, Diepgen TL. An open-label study assessing the safety and efficacy of alitretinoin in patients with severe chronic hand eczema unresponsive to topical corticosteroids. Clin Exp Dermatol. 2011;36:149-154.

18. Diepgen TL, Pfarr E, Zimmermann T. Efficacy and tolerability of alitretinoin for chronic hand eczema under daily practice conditions: results of the TOCCATA open study comprising 680 patients. Acta Derm Venereol. 2012;92:251-255.

19. National Institute for Health and Care Excellence. Eczema (chronic) - alitretinoin (TA177). 2009. Available from: http://www. nice.org.uk/TA177. Accessed February 27, 2014.

20. Irla N, Navarini AA, Yawalkar N. Alitretinoin abrogates innate inflammation in palmoplantar pustular psoriasis. Br J Dermatol. 2012 Nov;167(5):1170-1174.

21. Irla N, Navarini AA, Yawalkar N. Alitretinoin abrogates innate inflammation in palmoplantar pustular psoriasis. Br J Dermatol. 2012;167:1170-1174.

22. Coors EA, von den DP. Treatment of 2 patients with mycosis fungoides with alitretinoin. J Am Acad Dermatol. 2012;67:e265-e267.

23. Motschenbacher S, Buder K, Goebeler M, Kerstan A. Combined oral alitretinoin and narrowband UVB treatment of cutaneous $\mathrm{T}$ cell lymphoma: favourable response after multiple ineffective systemic therapies. Eur J Dermatol. 2012;22:803-804.

24. Molin S, Ruzicka T. Possible benefit of oral alitretinoin in T-lymphoproliferative diseases: a report of two patients with palmoplantar hyperkeratotic-rhagadiform skin changes and mycosis fungoides or Sézary syndrome. Br J Dermatol. 2009;161:1420-1422.
25. Kolios AG, Marques ME, Gubler C, et al. Oral, esophageal and cutaneous lichen ruber planus controlled with alitretinoin: case report and review of the literature. Dermatology. 2013;226:302-310.

26. Sárdy M, Ruzicka T. Successful therapy of refractory Hailey-Hailey disease with oral alitretinoin. Br J Dermatol. 2014;170:209-211.

27. Pampín A, Gómez-de la Fuente E, Caro Gutiérrez MD, LópezEstebaranz JL. Successful treatment of atypical adult pityriasis rubra pilaris with oral alitretinoin. J Am Acad Dermatol. 2013;69: e105-e106.

28. Letulé V, Herzinger T, Ruzicka T, Molin S. Treatment of Darier disease with oral alitretinoin. Clin Exp Dermatol. 2013;38:523-525.

29. Zamiri M, Munro CS. Successful treatment with oral alitretinoin in women of childbearing potential with Darier's disease. Br J Dermatol. 2013;169:709-710.

30. Kolesnik M, Gollnick H, Bonnekoh B. Complete remission of alopecia areata under treatment of chronic hand eczema with alitretinoin. Eur J Dermatol. 2013;23:110-111.

31. Prasad SC, Bygum A. Successful treatment with alitretinoin of dissecting cellulitis of the scalp in keratitis-ichthyosis-deafness syndrome. Acta Derm Venereol. 2013;93:473-474.

32. Schmitt-Hoffmann AH, Roos B, Sauer J, et al. Influence of alitretinoin on the pharmacokinetics of the oral contraceptive ethinyl estradiol/ norgestimate. Clin Exp Dermatol. 2011 Apr;36 Suppl 2:4-11.
Patient Preference and Adherence

\section{Publish your work in this journal}

Patient Preference and Adherence is an international, peer-reviewed, open access journal focusing on the growing importance of patient preference and adherence throughout the therapeutic continuum. Patient satisfaction, acceptability, quality of life, compliance, persistence and their role in developing new therapeutic modalities and compounds to

\section{Dovepress}

optimize clinical outcomes for existing disease states are major areas of interest. This journal has been accepted for indexing on PubMed Central. The manuscript management system is completely online and includes a very quick and fair peer-review system. Visit http://www.dovepress.com/ testimonials.php to read real quotes from published authors. 